MATEC Web of Conferences 11,01013 (2014)

DOI: $10.1051 /$ matecconf / 20141101013

(C) Owned by the authors, published by EDP Sciences, 2014

\title{
Effect of fillers Granulated slag on the durability of HPC in marine environment
}

\section{Effet de fillers de Laitier Granulé sur La Durabilité Des Bétons à Haute Performance En Milieu Marin}

\author{
Nadia Tebbal ${ }^{1}$ Zine El abidine Rahmouni ${ }^{2}$ et Zoubir Djendi ${ }^{2}$ \\ ${ }^{1}$ Université de M'sila, Institut de la gestion des techniques urbaines, Algérie \\ ${ }^{2}$ Université de M'sila, Department de Genie Civil, Algérie
}

\begin{abstract}
The combined effect of fillers of silica fume and blast furnace slag on the durability of high performance concretes was studied .The storage media are: seawater and tap water as reference. This work focuses on the effect of the incorporation of different amounts of milk more silica fume on the mechanical strength of concretes with limestone cements and its influence on sustainability with respect to marine. The results show that the addition of quantity $10 \%$ slag and $5 \%$ silica fume offers the best protection in marine waters .One of the indicators of sustainability are selected and monitored from 1 day to 28 days are variations of unconfined compressive strengths. The $\mathrm{PH}$ of the solution, especially related to the frequency of renewal baths, appears as an important parameter of aggressiveness.
\end{abstract}

Key-words. HPC, Marine environment, Silica fume, Granulated slag, Durability, Compressive strengths.

Résumé. L'effet conjugué des fillers de fumée silice et de laitier de haut fourneau sur la durabilité des bétons à hautes performances a été étudié. Les milieux de conservation sont : l'eau de mer et l'eau de robinet servant de référence. Le présent travail porte sur l'effet de l'incorporation de différentes quantités de laitier plus la fumée silice sur les résistances mécaniques des bétons formulés avec des ciments à base du calcaire et son influence sur la durabilité vis-à-vis des milieux marins. Les résultats montrent que l'ajout d'une quantité $(10 \%$ de laitier et $5 \%$ de la fumée de silice présente la meilleure protection dans les eaux marines. L'un des indicateurs de durabilités choisis et suivis de 1 jours à $28 \mathrm{j}$ sont les variations des résistances mécaniques en compression simple. Le PH des milieux, liés notamment à la fréquence de renouvellement des bains, apparaît comme un paramètre important d'agressivité.

Mots clés. BHP, Milieu marin, Fumée silice, Laitier granulé, Durabilité, Résistance mécanique 


\section{Introduction}

L'étude de la composition d'un béton consiste à définir le mélange optimal des différents granulats dont on dispose ainsi que le dosage en ciment et en eau afin de réaliser un béton dont les qualités soient celles recherchées pour la construction de l'ouvrage.

Le béton à haute résistance, ou plus généralement le béton à hautes performances, se compose de granulats, d'eau, de ciment, de superplastifiants, et éventuellement d'une addition (souvent, des fumées de silice et de laitier). L'utilisation des ajouts minéraux en remplacement du ciment est très importante, car en plus faire des économies d'énergie et la diminution de l'émission du $\mathrm{CO} 2$, tout en améliorant les propriétés du béton.

Le laitier des hauts fourneaux est l'ajout le plus connu et le plus fiable pour sa qualité. Cette étude consiste à formuler des bétons à hautes performances (BHP) à base de fillers de laitier granulé

La méthodologie de notre étude est basée sur l'optimisation des paramètres suivants : Le taux de fine d'addition et la formulation des BHP c.-à-d. La substitution d'additifs minéraux (fumé de silice) par des déchets industriels (laitier granulé des hauts fourneaux) provenant du complexe métallurgique (Elhadjar) d'Annaba. Le béton ainsi formulé est testé à l'état frais et à l'état durcis.

\section{Matériaux utilises et mode opératoire}

Le ciment : c'est un ciment Portland artificiel, CPACEM I 42,5 (CRS) de la cimenterie de ACC (M'sila). Il à une surface spécifique Blaine de $3200 \mathrm{~cm}^{2} / \mathrm{g}$ et une densité de $(3,1)$.

Le laitier granulé du haut fourneau : provient du complexe métallurgique (El-hadjar) d'Annaba,

Le sable : c'est un sable fin provenant de la région de Boussaâda (M'sila).

Tableau1. La composition chimique du ciment et du laitier utilisé dans cette étude est donnée au tableau 1.

\begin{tabular}{|c|c|c|c|}
\hline \multirow{4}{*}{$\begin{array}{c}\text { Composition } \\
\text { chimiques }\end{array}$} & $\begin{array}{c}\text { Compositions } \\
(\%)\end{array}$ & $\begin{array}{c}\text { Laitier } \\
\text { granulé }\end{array}$ & Ciment \\
\cline { 2 - 4 } & $\mathrm{SiO}_{2}$ & 38.9 & 21.66 \\
\cline { 2 - 4 } & $\mathrm{CaO}$ & 39.21 & 63.7 \\
\cline { 2 - 4 } & $\mathrm{Al}_{2} \mathrm{O}_{3}$ & 8.98 & 4.09 \\
\cline { 2 - 3 } & $\mathrm{FeO}_{3}$ & 0.95 & 4.54 \\
\cline { 2 - 3 } & $\mathrm{MgO}$ & 8.59 & 1.6 \\
\cline { 2 - 3 } & $\mathrm{SO}$ & 0.07 & 1.9 \\
\cline { 2 - 3 } & $\mathrm{MnO}$ & 1.17 & $/ / /$ \\
\hline \multirow{4}{*}{$\begin{array}{c}\text { Compositions } \\
\text { minéralogiques }\end{array}$} & $\mathrm{C} 3 \mathrm{~S}$ & 55 & \\
\cline { 2 - 3 } & $\mathrm{C} 2 \mathrm{~S}$ & 17 & \\
\cline { 2 - 3 } & $\mathrm{C} 3 \mathrm{~A}$ & 2.8 & \\
\cline { 2 - 3 } & $\mathrm{C} 4 \mathrm{AF}$ & 13 & \\
\cline { 2 - 3 } & \multicolumn{2}{|c}{13} \\
\hline
\end{tabular}

Le super plastifiant utilisé est le MEDAFLOW (40HR) (produit GRANITEX) présentant une densité de 1.1,

Les granulats : pour la confection des bétons dans cette recherche sont : un gravier (G1) de classe granulaire 3/8 $\mathrm{mm}$, un gravier (G2) de classe granulaire $8 / 15$, un sable siliceux naturel $(0 / 5)$ avec un module de finesse $M F 1=2.12$. On n'a pas pris en considération la nature et les propriétés mécaniques des granulats utilisés.

Fumée de silice (MEDAPLAST HP) de densité égale à 2.2 .

L'eau de gâchage utilisée c'est du robinet.

\section{Programme expérimental}

Le laitier est ajouté à raison de $8 \%$ et $25 \%$ de la masse du ciment, la fumée de silice $2 \%, 5 \%$ et $8 \%$. Les fillers utilisés sont broyés à une finesse de mouture mesurée sur tamis $80 \%$ égale à zéro, leurs dosages en sont ajustés de façon à obtenir une résistance en compression à 28 jours maximale pour une ouvrabilité acceptable.

pour obtenir un BHP on utilisant la méthode de formulation de l'université de Sherbrooke, qui permet de formuler des BHP sans air entraîné conformément à la norme ACI 211-1[Aitcin 2001].

Les compositions des bétons avec et sans pouzzolane retenues pour le programme expérimental sont reportées dans le tableau 2.

Tableau 2. Composition des bétons

\begin{tabular}{|c|c|c|c|c|c|}
\hline $\mathrm{kg} / \mathrm{m}^{3}$ & $\begin{array}{c}\mathrm{B} \\
(2 \mathrm{~F})\end{array}$ & $\begin{array}{c}\mathrm{B} \\
(5 \mathrm{~F})\end{array}$ & $\begin{array}{c}\mathrm{B} \\
(10 \mathrm{~F})\end{array}$ & \begin{tabular}{|c|}
$\mathrm{B}$ \\
$(2 \mathrm{~F}$ \\
$25 \mathrm{~L})$
\end{tabular} & $\begin{array}{c}\mathrm{B} \\
(5 \mathrm{~F} \\
10 \mathrm{~L})\end{array}$ \\
\hline $\begin{array}{l}\text { Sable de } \\
\text { dune SD }\end{array}$ & 711.6 & 707.1 & 501 & 695.6 & 700.8 \\
\hline Graviers 3/8 & \multicolumn{5}{|c|}{157.5} \\
\hline $\begin{array}{c}\text { Graviers } \\
8 / 15 \\
\end{array}$ & \multicolumn{5}{|c|}{892.5} \\
\hline Ciment C & 460.6 & 446.5 & 432.4 & 343.1 & 400 \\
\hline $\begin{array}{l}\text { Fumé de } \\
\text { silice }\end{array}$ & 9.212 & 23.03 & 46.06 & 9.21 & 23.03 \\
\hline Laitier & $/ / /$ & $/ / 1$ & $/ / / /$ & 115 & 46.06 \\
\hline $\mathrm{E} / \mathrm{L}$ & \multicolumn{5}{|c|}{0.3} \\
\hline Superpla & \multicolumn{5}{|c|}{4.41} \\
\hline
\end{tabular}

Dans tout ce qui suit la légende suivante a été adopté :

- B (2F), B (5F), B (10F) : Béton de fumée de silice à des dosages $(2 \%, 5 \%, 8 \%)$.

- $\mathrm{B}(2 \mathrm{~F}, 25 \mathrm{~L})$ : Béton dosé à $2 \%$ de fumée de silice et $25 \%$ de laitier

- $\mathrm{B}(5 \mathrm{~F}, 10 \mathrm{~L})$ : Béton dosé à $5 \%$ de fumée de silice et $10 \%$ de laitier. 
Les différentes éprouvettes de béton ainsi préparées sont conservées en salle humide $\left(20^{\circ} \mathrm{C}, \mathrm{m}\right.$ $5^{\circ} \mathrm{C}$ ) pendant $24 \mathrm{~h}$. Elles sont ensuite mises dans les différents bains de conservation [un milieu marin et un à eau potable] jusqu'à la réalisation des essais.

\section{Résultats et interprétations}

Pour toutes les gâchées des bétons réalisés, la maniabilité a été mesurée par l'affaissement au cône

d'ABRAMS conformément à la norme NF EN 12350-2. La résistance en compression axiale testée après 28 jours de conservation dans les deux milieux a été réalisée sur 3 éprouvettes cylindriques $110 \times 220 \mathrm{~mm}$ selon la norme NF EN 12390-4.

Les essais de traction par flexion pour tous les bétons ont été réalisés sur des éprouvettes prismatiques de dimension 100x100x400 $\mathrm{mm}$ après 28 jours de conservation selon la norme NF EN 12390-5. L'ensemble

des valeurs moyennes des résistances en compression axiale et en traction par flexion sont représentées dans les figures ci-après.

- Le nombre d'éprouvettes testés pour chaque essais de compression et traction : 6 éprouvettes pour chaque formulation .

\subsection{Optimisation du squelette granulaire (les ajouts cimentaires)}
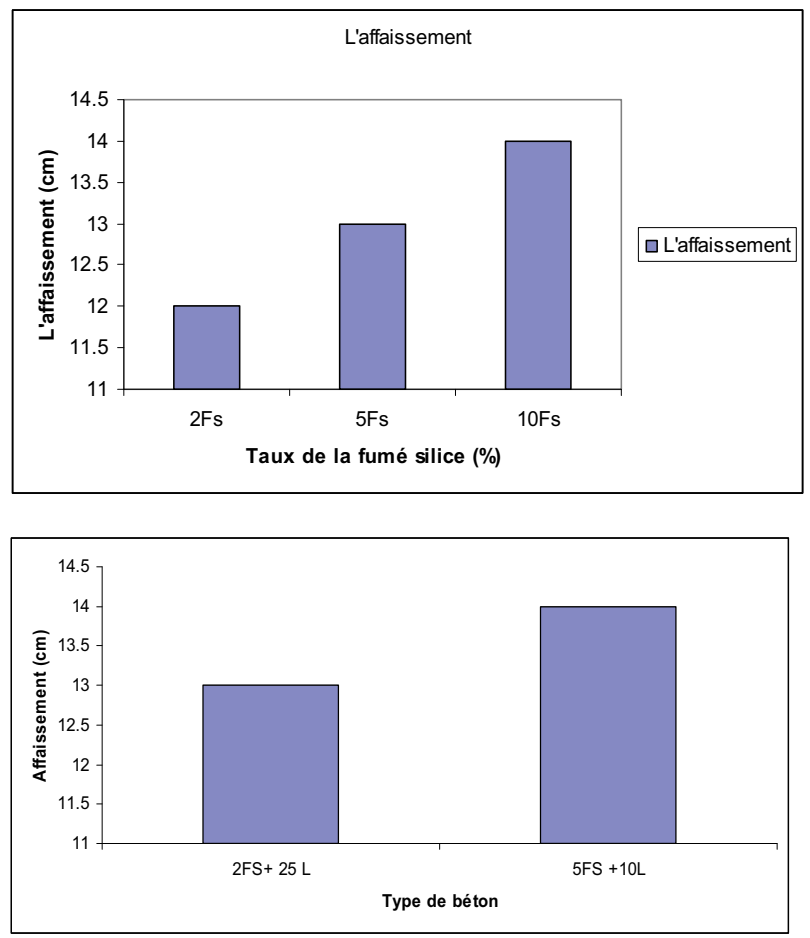

Fig 1. Affaissement au cône d'Abrams
On remarque d'après la figure 1 , un grand affaissement au cône d'Abrams qui augmente à chaque fois qu'on ajoute de l'ajout cimentaire.

\subsection{Résistance mécanique}
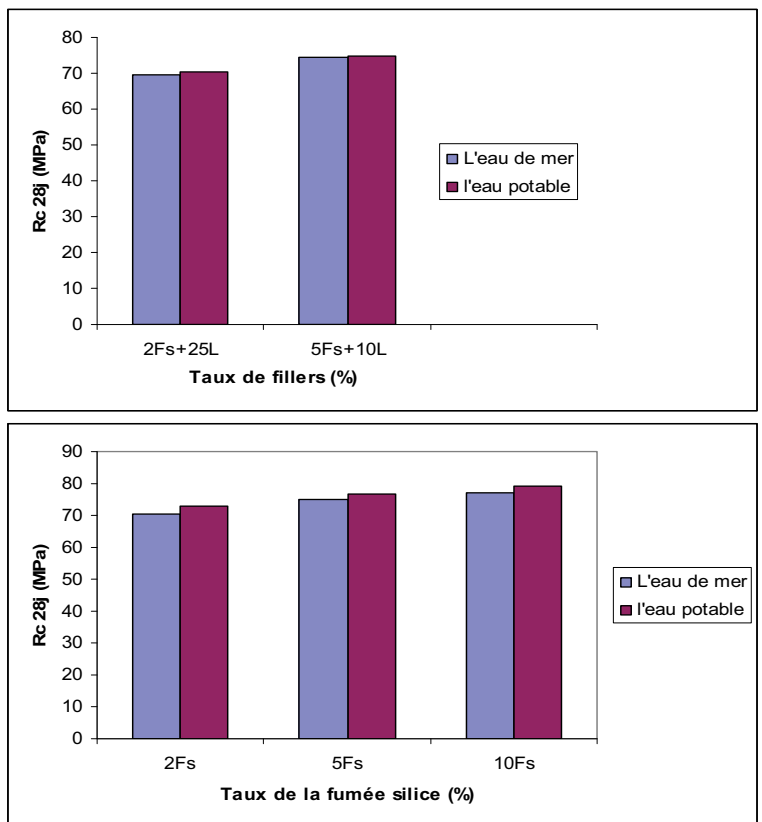

Fig 2 . Résistance à la compression des bétons
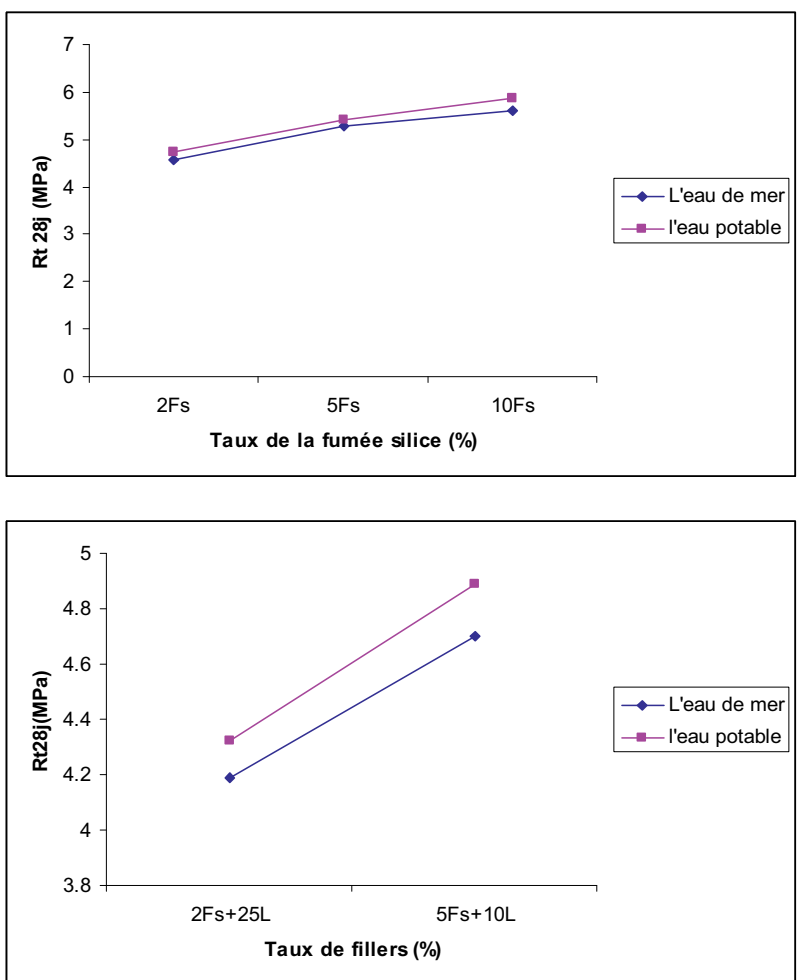

Fig 3 . Résistance à la traction des bétons 
résistances très élevées et qui dépassent les $75 \mathrm{MPa}$ à l'âge de 28j.

- D'après les figures 2 et 3 présentant l'évolution des résistances mécaniques des bétons testés, on remarque une amélioration de la résistance à la compression et à la traction du béton de pouzzolane. A $28 \mathrm{j}$ de conservation, on constate un gain de résistance en compression de l'ordre de $8 \%$ avec l'augmentation de taux de fumée de silice et un gain de $7 \%$ a celle de l'ajout laitier plus fumé de silice. Ce résultat était prévisible connaissant le rôle bénéfique de l'utilisation simultanée de la pouzzolane et d'un super plastifiant réducteur d'eau.

- L'introduction du laitier broyé augmente la maniabilité du béton mais donne des résistances à la compression et à la traction à court terme inférieures à celles du béton avec la même formulation et avec ajout du fumée de silice, par contre, donne une bonne résistance qui dépasse les $75 \mathrm{MPa}$ pour la formulation $\mathrm{B}(5 \mathrm{~F}, 10 \mathrm{~L})$ à 28 jours, cette résistance diminue avec l'augmentation du dosage en laitier broyé ; donc le dosage optimal en laitiers granulé broyé est inférieur à $150 \mathrm{~kg} / \mathrm{m}^{3}$ ce qui confirme les résultats de R.Chaid (2009).

- Dans notre cas on va considérer le dosage optimal en laitier égale à $100 \mathrm{~kg} / \mathrm{m}^{3}$ parce que la détermination $\mathrm{du}$ dosage optimal exacte nécessite beaucoup de temps et plusieurs tentatives.

- Les bétons conservés dans le milieu non agressif présentent des résistances meilleures à celle des bétons conservés dans le milieu agressif marin.

- L'effet de milieu marin peut être caractérisé par la diminution relative de la résistance en compression à $28 \mathrm{j}$ entre toutes les séries des bétons confectionnés.

- L'introduction du laitier avec la fumée de silice conduit à une croissance appréciable des résistances, cela est dû évidemment à son double rôle :

1. Densification de la matrice par effet filler, grâce à la petitesse de ses particules ultrafines qui s'intercalent dans les pores créés par l'hydratation du ciment et entre le granulat et les produits d'hydratation (auréole de transition).

2. Précipitation des C-S-H de deuxième génération due à l'effet pouzzolanique, résultat de l'interaction de l'hydroxyde de calcium $\mathrm{Ca}(\mathrm{OH}) 2$ libéré lors des réactions d'hydratation et les composants actifs de l'ajout.

- Les résultats obtenus dans cette phase montrent qu'il ya une amélioration de la résistance du béton pour l'ajout de $5 \%$ de fumée de silice et de $10 \%$ de laitier granulé broyé, car on a pu atteindre des

\section{Conclusions}

Les résultats obtenus dans cette étude nous ont permis d'aboutir aux conclusions suivantes :

- L'analyse des matériaux locaux montre qu'ils répondent aux normes pour la fabrication d'un béton de qualité.

- Le laitier granulé de haut fourneau du complexe sidérurgique de l'usine d'EL HADJAR, broyé (sous forme de fillers) convient pour la confection des bétons à hautes performances. Laitier broyé et la fumée de silice offrent des maniabilités acceptables malgré la rétention d'eau par le laitier granulé qui est assez importante en raison de leur porosité .Cette résultats observé par Laurent molez ,I. Mesoudene (2012).

- Les résultats obtenus sur béton frais et durci prouvent que le type des ajouts et leurs pourcentages ont une influence. Pour le béton B (10F) la diminution de la résistance à la compression de l'ordre de $26 \%$ par rapport au même pourcentage de fumée de silice. Influence aussi bien la résistance en compression à $28 \mathrm{j}$ que l'ouvrabilité. Le choix s'est donc porté sur $5 \%$ de fumée de silice et $25 \%$ de laitier.

- Pour le béton B $(2 \mathrm{~F}, 25 \mathrm{~L})$ la diminution de la résistance à la compression à $28 \mathrm{j}$ atteint $8 \%$ à celle du béton $\mathrm{B}(5 \mathrm{~F}$, $10 \mathrm{~L})$. Le choix s'est donc porté sur $5 \%$ de fumée de silice et $10 \%$ de laitier.

- Les bétons conservés dans un milieu non agressif présentent des résistances meilleures à celle des bétons conservés dans le milieu marin,

- La méthode de formulation des BHP de l'université de Sherbrooke est applicable pour la confection des BHP à base du laitier granulé broyé et donne de bons résultats.

\section{Référence bibliographique}

[1] Benslafa F. et Kazi A., Influence des paramètres de compositions sur les caractéristiques mécaniques d'un BHP, 1st International Conference on Sustainable Built Environnement Infrastructures in Developing Countries, ENSET Oran (Algeria) Octobre 12-14, 2009

[2] R. Chaid, Endommagement, déformations et microstructure des BHP de pouzzolane, 1st International Conférence on Sustainable Built 
environnement Infrastructures in Developing Countries, ENSET Oran (Algeria) - Octobre 12-14, 2009

[3] R. Lakhal1, Elaboration des bétons a hautes performances a base de laitier granulé, 1st International Conference on Sustainable Built environment Infrastructures in Developing Countries, ENSET Oran (Algeria) - October 12-14, 2009

[4] R. CHAID, Etude expérimentale de la durabilité des BHP aux ajouts cimentaires locaux Université Mentouri, Constantine, Algérie, 2008.

[5] Aitcin CP. 1995, Development in the application of high - performance concretes, construction and building materials, vol.9, No: 1, pp.13-17

[6] Laurent molez ,I. Mesoudene.2012, Effet des fillers de laitier et marbre sur la durabilité des ciments Portland dans des environnements de sulfate ; XXXe

Rencontres AUGC-IBPSA Chambéry, Savoie, 6 au 8 juin 2012 